\title{
Publisher's Correction to: Journal of the Korean Physical Society, Vol. 71, No. 10
}

[J. Korean Phys. Soc. 71, 593 (2017)], [J. Korean Phys. Soc. 71, 608 (2017)], [J. Korean Phys. Soc. 71, 628 (2017)], [J. Korean Phys. Soc. 71, 636 (2017)], [J. Korean Phys. Soc. 71, 647 (2017)], [J. Korean Phys. Soc. 71, 652 (2017)], [J. Korean Phys. Soc. 71, 657 (2017)], [J. Korean Phys. Soc. 71, 665 (2017)],

[J. Korean Phys. Soc. 71, 670 (2017)], [J. Korean Phys. Soc. 71, 679 (2017)], [J. Korean Phys. Soc. 71, 684 (2017)], [J. Korean Phys. Soc. 71, 692 (2017)], [J. Korean Phys. Soc. 71, 697 (2017)], [J. Korean Phys. Soc. 71, 701 (2017)], [J. Korean Phys. Soc. 71, 707 (2017)], [J. Korean Phys. Soc. 71, 711 (2017)],

[J. Korean Phys. Soc. 71, 717 (2017)], [J. Korean Phys. Soc. 71, 722 (2017)], [J. Korean Phys. Soc. 71, 727 (2017)], [J. Korean Phys. Soc. 71, 733 (2017)] and [J. Korean Phys. Soc. 71, 740 (2017)]

DOI: $10.3938 /$ jkps.71.1075

$$
\begin{array}{r}
\text { DOI } 10.3938 / \mathrm{jkps} .71 .593 \\
10.3938 / \mathrm{jkps} .71 .608 \\
10.3938 / \mathrm{jkps} .71 .628 \\
10.3938 / \mathrm{jkps} .71 .636 \\
10.3938 / \mathrm{jkps} .71 .647 \\
10.3938 / \mathrm{jkps} .71 .652 \\
10.3938 / \mathrm{jkps} .71 .657 \\
10.3938 / \mathrm{jkps} .71 .665 \\
10.3938 / \mathrm{jkps} .71 .670 \\
10.3938 / \mathrm{jkps} .71 .679 \\
10.3938 / \mathrm{jkps} .71 .684 \\
10.3938 / \mathrm{jkps} .71 .692 \\
10.3938 / \mathrm{jkps} .71 .697 \\
10.3938 / \mathrm{jkps} .71 .701 \\
10.3938 / \mathrm{jkps} .71 .707 \\
10.3938 / \mathrm{jkps} .71 .711 \\
10.3938 / \mathrm{jkps} .71 .717 \\
10.3938 / \mathrm{jkps} .71 .722 \\
10.3938 / \mathrm{jkps} .71 .727 \\
10.3938 / \mathrm{jkps} .71 .733 \\
10.3938 / \mathrm{jkps} .71 .740
\end{array}
$$

Regrettably, due to a technical error during the production process, there were discrepancies in DOI of the mentioned articles between HTML and PDF files. The DOIs are correct in the PDF files but were incorrect in HTML. The original articles have been corrected. The Publisher apologizes for any inconvenience and confusion caused. 